1 Hacettepe Journal of Mathematics and Statistics

h Volume 47 (2) (2018), 287-297

\title{
An existence and uniqueness result for linear fractional impulsive boundary value problems as an application of Lyapunov type inequality
}

\author{
Zeynep Kayar*
}

\begin{abstract}
A new and different approach to the investigation of the existence and uniqueness of solution of nonhomogenous impulsive boundary value problems involving the Caputo fractional derivative of order $\alpha(1<$ $\alpha \leq 2)$ is brought by using Lyapunov type inequality. To express and to analyze the unique solution, Green's function and its bounds are established, respectively. As far as we know, this approach based on the link between fractional boundary value problems and Lyapunov type inequality, has not been revealed even in the absence of impulse effect. Besides, the novel Lyapunov type inequality generalizes the related ones in the literature.
\end{abstract}

Keywords: Linear impulsive fractional boundary value problems, Green's function, Lyapunov type inequality, Disconjugacy.

2000 AMS Classification: 34A08, 34A37, 34B37, 34B27, 34C10

Received : 06.08.2016 Accepted : 02.02.2017 Doi : 10.15672/HJMS.2017.463

\section{Introduction}

In this paper we will give a sufficient condition for the existence and uniqueness of the solution in $P L C^{1}[a, b]=\left\{y:[a, b] \rightarrow \mathbb{R}: y^{\prime} \in P L C[a, b]\right\}$, where

$P L C[a, b]=\left\{y:[a, b] \rightarrow \mathbb{R}\right.$ is continuous on each interval $\left(\tau_{i}, \tau_{i+1}\right)$, the limits $y\left(\tau_{i}^{ \pm}\right)$ exist and $y\left(\tau_{i}^{-}\right)=y\left(\tau_{i}\right)$ for $\left.i=1,2, \ldots, p\right\}$, for the linear impulsive nonhomogenous fractional boundary value problem

*Van Yüzüncü Yll University, Department of Mathematics, 65080, Van, Turkey, Email: zeynepkayar@yyu.edu.tr 


$$
\begin{gathered}
\left({ }_{a}^{C} D^{\alpha} y\right)(t)+f(t) y(t)=g(t), \quad t \neq \tau_{i}, \quad a<t<b, \quad 1<\alpha \leq 2, \\
\left.\Delta y\right|_{t=\tau_{i}}=a_{i}, \quad i=1,2, \ldots, p, \\
\left.\Delta y^{\prime}\right|_{t=\tau_{i}}=-\frac{\gamma_{i}}{\beta_{i}} y\left(\tau_{i}^{-}\right)+b_{i}, \quad i=1,2, \ldots, p, \\
y(a)=A, y(b)=B,
\end{gathered}
$$

by showing nonexistence of nontrivial solution of the corresponding impulsive homogenous fractional boundary value problem

$$
\begin{gathered}
\left({ }_{a}^{C} D^{\alpha} y\right)(t)+f(t) y(t)=0, \quad t \neq \tau_{i}, \quad a<t<b, \quad 1<\alpha \leq 2, \\
\left.\Delta y\right|_{t=\tau_{i}}=0, \quad i=1,2, \ldots, p \\
\left.\Delta y^{\prime}\right|_{t=\tau_{i}}=-\frac{\gamma_{i}}{\beta_{i}} y\left(\tau_{i}^{-}\right), \quad i=1,2, \ldots, p \\
y(a)=0, y(b)=0,
\end{gathered}
$$

where ${ }_{a}^{C} D^{\alpha}$ is Caputo fractional derivative of order $\alpha(1<\alpha \leq 2)$, $f, g: P L C[a, b] \rightarrow \mathbb{R}$ are given functions and $a, b, A, B$ are given real constants. The impulse condition is given by delta operator which is defined as $\left.\Delta y\right|_{t=\tau_{i}}=y\left(\tau_{i}^{+}\right)-y\left(\tau_{i}^{-}\right)=y\left(\tau_{i}^{+}\right)-y\left(\tau_{i}\right)$. For convention let us choose $a=\tau_{0}<\tau_{1}<\ldots<\tau_{p}<\tau_{p+1}=b$.

It should be noted that since $\left.\Delta y\right|_{t=\tau_{i}}=0$, homogenous boundary value problem (1.2a)-(1.2d) has continuos solutions. On the other hand, the main theorem of this paper can also be applicable to the following impulsive homogenous fractional boundary value problem

$$
\begin{array}{cl}
\left({ }_{a}^{C} D^{\alpha} u\right)(t)+f(t) u(t)=0, & t \neq \tau_{i}, \quad a<t<b, \quad 1<\alpha \leq 2, \\
u\left(\tau_{i}^{+}\right)=\beta_{i} u\left(\tau_{i}^{-}\right), & i=1,2, \ldots, p, \\
u^{\prime}\left(\tau_{i}^{+}\right)=\beta_{i} u^{\prime}\left(\tau_{i}^{-}\right)-\gamma_{i} u\left(\tau_{i}^{-}\right), & i=1,2, \ldots, p, \\
u(a)=0, u(b)=0, &
\end{array}
$$

with discontinuos solution. Indeed, if we define

$$
y(t)=\frac{u(t)}{\beta_{1} \beta_{2} \ldots \beta_{i}}, t \in\left(\tau_{i}, \tau_{i+1}\right), y\left(\tau_{i}\right)=y\left(\tau_{i}^{-}\right) \text {, then (1.3a)-(1.3d) becomes as ho- }
$$
mogenous boundary value problem $(1.2 \mathrm{a})-(1.2 \mathrm{~d})$.

Mathematical description and modelling of many engineering and scientific problems, which have memory and hereditary properties, by using fractional differential equations is more adequate than by using ordinary differential equations due to the fact that there are more degrees of freedom in the fractional-order models. Therefore fractional-order models become more natural and useful than the classical integer-order models. For this reason fractional differential equations arise in many engineering and scientific disciplines such as physics, chemistry, biology, economics, control theory, signal and image processing, biophysics, blood flow phenomena, aerodynamics, fitting of experimental data, see [26, 28, $22,30]$ and the references therein. Investigation of the theory of integer order differential equations under impulse effect has developed rapidly in the last three decades [23, 8 , $31,7]$ because they are not only one of the fundamental problems in most branches of applied mathematics, science and technology but also used to describe the dynamics of processes in which sudden, discontinuous jumps occur, such as harvesting, earthquakes, diseases, and so forth. Since boundary value problems involving the Caputo fractional derivative of order $\alpha(1<\alpha \leq 2)$ play an important role in theory and applications, 
there are many papers on nonimpulsive case $[37,2,4,5,35]$ and on the impulsive case $[6,32,3,38,34,39,36,40,41,42]$ based on different fixed point theorems. However, showing the existence and uniqueness of solution to the fractional impulsive boundary value problems by using Lyapunov type inequality has not been considered till now. The method given for the first time in [21] arised from the connection of nonhomogenous boundary value problems and Lyapunov type inequality. To the best of our knowledge, this connection appears for fractional impulsive boundary value problems for the first time and has not been noticed even for the nonimpulsive case.

In a celebrated paper of 1893, Lyapunov [25] prove the following result.

1.1. Theorem ([25]). If the boundary value problem

$$
\begin{aligned}
& y^{\prime \prime}+q(t) y=0, \quad a<t<b, \\
& y(a)=y(b)=0
\end{aligned}
$$

has a nontrivial solution, where $q$ is a real and continuous function with $q(t) \geq 0, q(t) \not \equiv 0$, then the so-called Lyapunov inequality

$$
\int_{a}^{b} q(t) d t>\frac{4}{b-a}
$$

holds.

After the initiated work of Lyapunov [25], many authors have paid a considerable attention to Lyapunov type inequalities and various proofs and generalizations or improvements have appeared in the literature. For a comprehensive exibition of these results we refer two surveys [10,33] and references therein. The result for (1.4) in [9] is worth mentioning due to its contribution to this subject. Borg [9] changed the nonnegativity condition of $q(t)$ by nonnegative integral of $q(t)$ and improved inequality (1.5).

1.2. Theorem ([9]). If the boundary value problem (1.4) has a nontrivial solution, where $q$ is a real and continuous function with $q(t) \not \equiv 0$, then we have the Lyapunov type inequality

$$
\int_{a}^{b}|q(t)| d t>\frac{4}{b-a}
$$

The second-order differential equations under impulse effect

$$
\begin{array}{ll}
\left(p(t) y^{\prime}\right)^{\prime}+q(t) y=0, & t \neq \tau_{i} \\
y\left(\tau_{i}^{+}\right)=\beta_{i} y\left(\tau_{i}^{-}\right), \quad\left(p y^{\prime}\right)\left(\tau_{i}^{+}\right)=-\gamma_{i} y\left(\tau_{i}^{-}\right)+\beta_{i}\left(p y^{\prime}\right)\left(\tau_{i}^{-}\right), & i \in \mathbb{N} .
\end{array}
$$

was considered first in [15] and the extended Lyapunov-type inequality is given therein by using modified definition of zero of a function. For piecewise defined functions, the concept of a zero of a function is replaced by a so-called generalized zero.

1.3. Definition ( $[17,15,21])$. A real number $c$ is called a zero (generalized zero) of a function $f$ if and only if $f\left(c^{-}\right)=0$ or $f\left(c^{+}\right)=0$. If $f$ is continuous function at $c$, then $c$ becomes a real zero.

1.4. Theorem ([15]). Let $p(t)>0$ and $\beta_{i} \neq 0$ for $i \in \mathbb{N}$. If $y(t)$ is a nontrivial solution of (1.7) with $y\left(a^{+}\right)=0=y\left(b^{-}\right)$, where $a, b \in \mathbb{R}$ with $a<b$ and $y(t) \neq 0$ for $t \in(a, b)$, then we have the Lyapunov type inequality

$$
\left[\int_{a}^{b} \frac{1}{p(t)} d t\right]\left[\int_{a}^{b} q^{+}(t) d t+\sum_{\tau_{i} \in[a, b)}\left(\frac{\gamma_{i}}{\beta_{i}}\right)^{+}\right]>4,
$$

where $q^{+}(t)=\max \{q(t), 0\}$ and $\left(\frac{\gamma_{i}}{\beta_{i}}\right)^{+}=\max \left\{\frac{\gamma_{i}}{\beta_{i}}, 0\right\}$. 
For $\alpha \in(1,2]$, the fractional counterparts of Lyapunov type inequlity is obtained in $[13,14,29,18,19,20]$.

The theory of disconjugacy is well developed for ordinary differential equations, the history of which starts with [16, 17, 24, 11, 27]. However, generalization of this theory to the fractional case is not considered much, see [1, 12].

Motivated by the aforementioned works we have discussed the existence and uniqueness of solutions of fractional impulsive boundary value problems. In Section 2 we recall some preliminary facts that we will use in the sequel. Section 3 contains auxiliary tools, which are Green's function and its properties, Lyapunov type inequality and disconjugacy criterion, used to prove the main result. Section 4 is devoted to the main result, which is the existence and uniqueness theorem for fractional impulsive nonhomogenous boundary value problem (1.1a)-(1.1d). To the best of our knowledge although many results have been obtained for impulsive fractional boundary value problems by using different techniques, there is little known about the connection of fractional boundary value problems and Lyapunov type inequality even for nonimpulsive case.

\section{Preliminaries}

Before going further, let us start with basic definitions and some facts about RiemannLiouville fractional integral, Riemann-Liouville fractional derivative and Caputo fractional derivative and give definition of disconjugacy for fractional differential equations.

2.1. Definition. [26, 28, 22] Let $\alpha \geq 0$ and $\phi$ be a continuous function defined on $[a, b]$. The Riemann Lioville fractional integral of order $\alpha$ is defined by

$$
\left({ }_{a} I^{\alpha} \phi\right)(t)=\frac{1}{\Gamma(\alpha)} \int_{a}^{t}(t-s)^{\alpha-1} \phi(s) d s \text { for } \alpha>0
$$

and ${ }_{a} I^{0} \phi(t)=\phi(t)$ for $\alpha=0$.

2.2. Definition. [26, 28, 22] The Riemann Liouville fractional derivative of order $\alpha \geq 0$ is defined by

$$
\left({ }_{a} D^{\alpha} \phi\right)(t)= \begin{cases}\left({ }_{a} D^{m}{ }_{a} I^{m-\alpha} \phi\right)(t), & \alpha>0 \\ \phi(t), & \alpha=0\end{cases}
$$

where $m$ is the smallest integer greater or equal than $\alpha$.

2.3. Definition. $[26,28,22]$ The Caputo fractional derivative of order $\alpha \geq 0$ is defined by

$$
\left(\begin{array}{ll}
\left.{ }_{a}^{C} D^{\alpha} \phi\right)(t)= \begin{cases}\left({ }_{a} I^{m-\alpha}{ }_{a} D^{m} \phi\right)(t), & \alpha>0 \\
\phi(t), & \alpha=0\end{cases}
\end{array}\right.
$$

where $m$ is the smallest integer greater or equal than $\alpha$.

2.4. Lemma. [22] If $y(t) \in A C^{m}[a, b]$ or $y(t) \in C^{m}[a, b]$, then for some constants $c_{i}, i=1,2, \ldots, m$, one has

$$
{ }_{a} I^{\alpha}{ }_{a}^{C} D^{\alpha} \phi(t)=\phi(t)+c_{1}+c_{2}(t-a)+c_{2}(t-a)^{2} \ldots+c_{m}(t-a)^{m-1},
$$

where $m$ is the smallest integer greater or equal than $\alpha$.

2.5. Definition. Equation (1.2a)-(1.2c) is called disconjugate on an interval $[a, b]$ if and only if all solutions of equation (1.2a)-(1.2c) have at most one zero on the interval $[a, b]$. 


\section{Preparatory Theorems}

To obtain an existence uniqueness criterion, we need to establish some auxiliary results in a series of theorems. The first two theorems provide Green's function and its properties, the last two yield Lyapunov type inequality and disconjugacy criterion.

3.1. Green's function and its properties. In this section we will find Green's function to write the integral equation of the solution of the nonhomogenous problem (1.1a)(1.1d) .

3.1. Theorem. $y \in P L C^{1}[a, b]$ is a solution of the boundary value problem (1.1a)-(1.1d) if and only if $y$ satisfies the following integral equation

$$
\begin{aligned}
y(t) & =A+\frac{(t-a)(B-A)}{b-a}+\int_{a}^{b} G(t, s)[g(s)-f(s) y(s)] \\
& +\sum_{a \leq \tau_{i}<b} H\left(t, \tau_{i}\right)\left[b_{i}-\frac{\gamma_{i}}{\beta_{i}} y\left(\tau_{i}\right)\right]+\sum_{a \leq \tau_{i}<b} K\left(t, \tau_{i}\right) a_{i},
\end{aligned}
$$

where

$$
\begin{aligned}
& G(t, s)=\frac{1}{\Gamma(\alpha)} \begin{cases}\frac{a-t}{b-a}(b-s)^{\alpha-1}+(t-s)^{\alpha-1}, & a \leq s<t \leq b \\
\frac{a-t}{b-a}(b-s)^{\alpha-1}, & a \leq t \leq s \leq b\end{cases} \\
& H\left(t, \tau_{i}\right)= \begin{cases}\frac{\left(a-\tau_{i}\right)(b-t)}{b-a}, & a \leq \tau_{i}<t \leq b \\
\frac{a-t}{b-a}\left(b-\tau_{i}\right), & a \leq t \leq \tau_{i} \leq b\end{cases} \\
& K\left(t, \tau_{i}\right)= \begin{cases}\frac{b-t}{b-a}, & a \leq \tau_{i}<t \leq b \\
\frac{a-t}{b-a}, & a \leq t \leq \tau_{i} \leq b\end{cases}
\end{aligned}
$$

Proof. The proof is the generalization of the proof given in [14] but for the completeness of this paper, we will give all the proofs in detail.

It is known for Caputo fractional derivative that if $t \in\left[\tau_{p}, \tau_{p+1}\right]$, then $y$ is a solution of (1.1a) if and only if

$$
\begin{aligned}
y(t) & =c_{1}+c_{2} t+\frac{1}{\Gamma(\alpha)} \int_{a}^{t}(t-s)^{\alpha-1}[g(s)-f(s) y(s)] d s \\
& +\sum_{i=1}^{p} a_{i}+\sum_{i=1}^{p}\left(t-\tau_{i}\right)\left[b_{i}-\frac{\gamma_{i}}{\beta_{i}} y\left(\tau_{i}\right)\right]
\end{aligned}
$$

for some real constants $c_{1}, c_{2}$. The first boundary condition, $y(a)=A$ implies that

$$
c_{1}+c_{2} a=A \text {. }
$$

By imposing the second boundary condition, $y(b)=B$, we have

$$
\begin{aligned}
y(b)=y\left(\tau_{p+1}\right) & =c_{1}+c_{2} b+\frac{1}{\Gamma(\alpha)} \int_{a}^{b}(b-s)^{\alpha-1}[g(s)-f(s) y(s)] d s \\
& +\sum_{i=1}^{p} a_{i}+\sum_{i=1}^{p}\left(b-\tau_{i}\right)\left[b_{i}-\frac{\gamma_{i}}{\beta_{i}} y\left(\tau_{i}\right)\right]=B .
\end{aligned}
$$

Then we obtain 


$$
\begin{aligned}
c_{2} & =\frac{B-A}{b-a}-\frac{1}{\Gamma(\alpha)(b-a)} \int_{a}^{b}(b-s)^{\alpha-1}[g(s)-f(s) y(s)] d s \\
& -\frac{1}{b-a} \sum_{i=1}^{p} a_{i}-\frac{1}{b-a} \sum_{i=1}^{p}\left(b-\tau_{i}\right)\left[b_{i}-\frac{\gamma_{i}}{\beta_{i}} y\left(\tau_{i}\right)\right]
\end{aligned}
$$

and

$$
\begin{aligned}
c_{1} & =a \frac{A-B}{b-a}+\frac{a}{\Gamma(\alpha)(b-a)} \int_{a}^{b}(b-s)^{\alpha-1}[g(s)-f(s) y(s)] d s \\
& +\frac{a}{b-a} \sum_{i=1}^{p} a_{i}+\frac{a}{b-a} \sum_{i=1}^{p}\left(b-\tau_{i}\right)\left[b_{i}-\frac{\gamma_{i}}{\beta_{i}} y\left(\tau_{i}\right)\right]+A
\end{aligned}
$$

and hence for $t \in\left(\tau_{r}, \tau_{r+1}\right]$, where $a<\tau_{1}<\tau_{2}<\ldots<\tau_{r}<\tau_{r+1}<\ldots<\tau_{p}<b$,

$$
\begin{aligned}
y(t) & =A+\frac{(t-a)(B-A)}{b-a}+\frac{1}{\Gamma(\alpha)} \int_{a}^{b} \frac{a-t}{b-a}(b-s)^{\alpha-1}[g(s)-f(s) y(s)] d s \\
& +\frac{1}{\Gamma(\alpha)} \int_{a}^{t}(t-s)^{\alpha-1}[g(s)-f(s) y(s)] d s+\sum_{i=1}^{r} a_{i}+\sum_{i=1}^{p} \frac{a-t}{b-a} a_{i} \\
& +\sum_{i=1}^{p} \frac{a-t}{b-a}\left(b-\tau_{i}\right)\left[b_{i}-\frac{\gamma_{i}}{\beta_{i}} y\left(\tau_{i}\right)\right]+\sum_{i=1}^{r}\left(t-\tau_{i}\right)\left[b_{i}-\frac{\gamma_{i}}{\beta_{i}} y\left(\tau_{i}\right)\right],
\end{aligned}
$$

which implies the desired result.

The following theorem provides upper and lower bounds for Green's functions $G, H, K$ which will be used in the next section.

3.2. Theorem. Green's functons $G, H, K$ satisfy the following properties:

1) $|G(t, s)| \leq \frac{1}{\Gamma(\alpha)} \frac{(\alpha-1)^{\alpha-1}}{\alpha^{\alpha}}(b-a)^{\alpha-1}$ for all $a \leq t, s \leq b$.

2) $H\left(t, \tau_{i}\right) \leq 0$ and $\left|H\left(t, \tau_{i}\right)\right| \leq \frac{b-a}{4}$ for all $a \leq t, \tau_{i} \leq b$.

3) $\left|K\left(t, \tau_{i}\right)\right| \leq 1$ for all $a \leq t, \tau_{i} \leq b, i=1,2, \ldots, p$.

Proof. 1) By employing the same tools used in [14, Lemma 2], it can be shown that $|G(t, s)| \leq \frac{1}{\Gamma(\alpha)} \frac{(\alpha-1)^{\alpha-1}}{\alpha^{\alpha}}(b-a)^{\alpha-1}$.

2) Let us define two functions

$$
\begin{aligned}
& h_{1}\left(t, \tau_{i}\right)=\frac{\left(a-\tau_{i}\right)(b-t)}{b-a}, \quad a \leq \tau_{i}<t \leq b \text { and } \\
& h_{2}\left(t, \tau_{i}\right)=\frac{a-t}{b-a}\left(b-\tau_{i}\right), \quad a \leq t \leq \tau_{i} \leq b . \\
& \text { Then } \\
& \left|h_{1}\left(t, \tau_{i}\right)\right|=\frac{\left(\tau_{i}-a\right)(b-t)}{b-a} \leq \frac{(t-a)(b-t)}{b-a} \leq \frac{b-a}{4} \\
& \text { and } \\
& \left|h_{2}\left(t, \tau_{i}\right)\right|=\frac{t-a}{b-a}\left(b-\tau_{i}\right) \leq \frac{\tau_{i}-a}{b-a}\left(b-\tau_{i}\right) \leq \frac{b-a}{4}
\end{aligned}
$$

where we have used the inequality

$$
\frac{1}{y-x}+\frac{1}{z-y} \geq \frac{4}{z-x}
$$


for arbitrary real numbers $x, y, z$ satisfying $x<y<z$.

3) Since

$$
\begin{aligned}
& 0 \leq k_{1}\left(t, \tau_{i}\right)=\frac{b-t}{b-a} \leq \frac{b-a}{b-a}=1 \\
& \text { and } \\
& -1=\frac{a-b}{b-a} \leq k_{2}\left(t, \tau_{i}\right)=\frac{a-t}{b-a} \leq 0, \text { the third Green function } K\left(t, \tau_{i}\right) \text { satisfies }
\end{aligned}
$$
the property that $\left|K\left(t, \tau_{i}\right)\right| \leq 1$.

3.2. Lyapunov type inequality for homogenous problem. In order to show the uniqueness of the solutions of nonhomogenous boundary value problem (1.1a)-(1.1d) in the main theorem, Lyapunov type inequality and disconjugacy criterion for the corresponding homogenous boundary value problem (1.2a)-(1.2d) are established.

3.3. Theorem. If homogenous boundary value problem (1.2a)-(1.2d) has a nontrivial solution $y(t) \neq 0$ on $(a, b)$, then we have Lyapunov type inequality

$$
\int_{a}^{b}|f(s)| d s+\sum_{a \leq \tau_{i}<b}\left(\frac{\gamma_{i}}{\beta_{i}}\right)^{+}>\min \left\{\frac{4}{b-a}, \frac{\Gamma(\alpha) \alpha^{\alpha}}{[(\alpha-1)(b-a)]^{\alpha-1}}\right\},
$$

where $\left(\frac{\gamma_{i}}{\beta_{i}}\right)^{+}=\max \left\{\frac{\gamma_{i}}{\beta_{i}}, 0\right\}$.

Proof. Since $D^{\alpha}$ is a linear operator, without loss of generality we may assume that $y(t)>0$ on $(a, b)$. Since $y(t)$ is continuos on $[a, b]$, there exist a point $c$ in $[a, b]$ such that $\max _{t \in[a, b]} y(t)=y(c)$. Then by using (3.1), we obtain

$$
\begin{aligned}
y(c) & =-\int_{a}^{b} G(c, s) f(s) y(s)-\sum_{a \leq \tau_{i}<b} H\left(c, \tau_{i}\right) \frac{\gamma_{i}}{\beta_{i}} y\left(\tau_{i}\right) \\
& \leq \int_{a}^{b}|G(c, s)||f(s)| y(s)-\sum_{a \leq \tau_{i}<b} H\left(c, \tau_{i}\right)\left(\frac{\gamma_{i}}{\beta_{i}}\right)^{+} y\left(\tau_{i}\right),
\end{aligned}
$$

where $\left(\frac{\gamma_{i}}{\beta_{i}}\right)^{+}=\max \left\{\frac{\gamma_{i}}{\beta_{i}}, 0\right\}$. Since $y(t) \leq y(c)$ for all $t \in[a, b]$, we have

$$
y(c)<y(c) \int_{a}^{b}|G(c, s)||f(s)| d s-y(c) \sum_{a \leq \tau_{i}<b} H\left(c, \tau_{i}\right)\left(\frac{\gamma_{i}}{\beta_{i}}\right)^{+} .
$$

Employing the properties of Green's function, $G$ and $H$, inequality (3.7) turns into

$$
\begin{aligned}
1 & <\int_{a}^{b}|G(c, s)||f(s)| d s-\sum_{a \leq \tau_{i}<b} H\left(c, \tau_{i}\right)\left(\frac{\gamma_{i}}{\beta_{i}}\right)^{+} \\
& \leq \frac{1}{\Gamma(\alpha)} \frac{[(\alpha-1)(b-a)]^{\alpha-1}}{\alpha^{\alpha}} \int_{a}^{b}|f(s)| d s+\frac{b-a}{4} \sum_{a \leq \tau_{i}<b}\left(\frac{\gamma_{i}}{\beta_{i}}\right)^{+} \\
& \leq \max \left\{\frac{1}{\Gamma(\alpha)} \frac{[(\alpha-1)(b-a)]^{\alpha-1}}{\alpha^{\alpha}}, \frac{b-a}{4}\right\}\left[\int_{a}^{b}|f(s)| d s+\sum_{a \leq \tau_{i}<b}\left(\frac{\gamma_{i}}{\beta_{i}}\right)^{+}\right]
\end{aligned}
$$

which yields the desired result.

3.4. Remark. If $\alpha=2$, then fractional impulsive boundary value problem (1.2a)-(1.2d) becomes as impulsive boundary value problem involving integer order derivative considered in [15] with $p(t)=1$. Then inequality (3.5) reduces to inequality (1.8). 
3.5. Remark. If there is no impulse effect, then Theorem 2.3 reduces to [ 14 , Theorem 1]. Therefore inequality (3.5) is the impulsive generalization of inequality in [14].

3.6. Remark. If $\alpha=2$, and $\beta_{i}=1, \gamma_{i}=0$ for all $i=1,2, \ldots, p$, then homogenous fractional boundary value problem (1.2a)-(1.2b) reduces to boundary value problem (1.4) involving integer order derivative considered in [25] and [9]. Hence inequality (3.5) is the fractional generalization of inequality (1.6) and it is an extension and improvement of inequality (1.5) to the fractional case.

3.3. Disconjugacy criterion for homogenous problem. Since the sufficient condition for the uniqueness of solution of the boundary value problem (1.1a)-(1.1d) is obtained by disconjugacy criterion, in this section this criterion is established by using Lyapunov type inequality. Since Lyapunov inequality implies disconjugacy criterion directly, it can be considered as an application of Lyapunov type inequality.

\subsection{Theorem. If}

$$
\int_{a}^{b}|f(s)| d s+\sum_{a \leq \tau_{i}<b}\left(\frac{\gamma_{i}}{\beta_{i}}\right)^{+} \leq \min \left\{\frac{4}{b-a}, \frac{\Gamma(\alpha) \alpha^{\alpha}}{[(\alpha-1)(b-a)]^{\alpha-1}}\right\}
$$

where $\left(\frac{\gamma_{i}}{\beta_{i}}\right)^{+}=\max \left\{\frac{\gamma_{i}}{\beta_{i}}, 0\right\}$, then equation (1.2a)-(1.2c) is disconjugate on $[a, b]$.

Proof. Suppose on the contrary that equation $(1.2 \mathrm{a})-(1.2 \mathrm{c})$ is not disconjugate on $[a, b]$. Then there exist a nontrivial solution $y$ of equation $(1.2 \mathrm{a})-(1.2 \mathrm{c})$ and at least two points $t_{1}, t_{2} \in[a, b]$ such that $y\left(t_{1}\right)=y\left(t_{2}\right)=0$ for $t \in[a, b]$ and $y(t) \neq 0$ for $t \in[a, b]$. Then by using Lyapunov type inequality on the inerval $\left[t_{1}, t_{2}\right]$, we have

$$
\int_{t_{1}}^{t_{2}}|f(s)| d s+\sum_{t_{1} \leq \tau_{i}<t_{2}}\left(\frac{\gamma_{i}}{\beta_{i}}\right)^{+}>\min \left\{\frac{4}{t_{2}-t_{1}}, \frac{\Gamma(\alpha) \alpha^{\alpha}}{\left[(\alpha-1)\left(t_{2}-t_{1}\right)\right]^{\alpha-1}}\right\}
$$

and hence

$$
\begin{aligned}
\int_{a}^{b}|f(s)| d s+\sum_{a \leq \tau_{i}<b}\left(\frac{\gamma_{i}}{\beta_{i}}\right)^{+} & \geq \int_{t_{1}}^{t_{2}}|f(s)| d s+\sum_{t_{1} \leq \tau_{i}<t_{2}}\left(\frac{\gamma_{i}}{\beta_{i}}\right)^{+} \\
& >\min \left\{\frac{4}{t_{2}-t_{1}}, \frac{\Gamma(\alpha) \alpha^{\alpha}}{\left[(\alpha-1)\left(t_{2}-t_{1}\right)\right]^{\alpha-1}}\right\} \\
& \geq \min \left\{\frac{4}{b-a}, \frac{\Gamma(\alpha) \alpha^{\alpha}}{[(\alpha-1)(b-a)]^{\alpha-1}}\right\}
\end{aligned}
$$

which contradicts inequality (3.8)

\section{Main Result}

Existence and uniqueness result for the nonhomogenous boundary problem (1.1a)(1.1d) is given in the following theorem.

4.1. Theorem. If

$$
\int_{a}^{b}|f(s)| d s+\sum_{a \leq \tau_{i}<b}\left(\frac{\gamma_{i}}{\beta_{i}}\right)^{+} \leq \min \left\{\frac{4}{b-a}, \frac{\Gamma(\alpha) \alpha^{\alpha}}{[(\alpha-1)(b-a)]^{\alpha-1}}\right\}
$$

where $\left(\frac{\gamma_{i}}{\beta_{i}}\right)^{+}=\max \left\{\frac{\gamma_{i}}{\beta_{i}}, 0\right\}$, then nonhomogenous boundary problem (1.1a)-(1.1d) has a unique solution which is also a unique solution of integral equation (3.1). 
Proof. The proof is based on the arguments developed in [21]. It is shown in the proof of Theorem 3.1 that $y$ is the solution of nonhomogenous boundary problem (1.1) if and only if it is a solution of integral equation (3.1). To prove the uniqueness, it is sufficient to show that the homogenous boundary value problem $(1.2 \mathrm{a})-(1.2 \mathrm{~d})$ has only trivial solution. Assume on the contrary that $y(t) \not \equiv 0$ is a solution of the homogenous boundary value problem $(1.2 \mathrm{a})-(1.2 \mathrm{~d})$. Then by using Lyapunov type inequality, we have

$$
\int_{a}^{b}|f(s)| d s+\sum_{a \leq \tau_{i}<b}\left(\frac{\gamma_{i}}{\beta_{i}}\right)^{+}>\min \left\{\frac{4}{b-a}, \frac{\Gamma(\alpha) \alpha^{\alpha}}{[(\alpha-1)(b-a)]^{\alpha-1}}\right\}
$$

which gives a contradiction to (4.1). Therefore the homogenous boundary value problem (1.2a)-(1.2d) has only trivial solution. Due to the theory of linear fractional boundary value problems, the nonhomogenous boundary problem (1.1a)-(1.1d) has a unique solution.

\section{References}

[1] Abdeljawad, T. and Baleanu, D. and Jarad, F. and Mustafa, O. G. and Trujillo, J. J. A Fite type result for sequential fractional differential equations, Dynam. Systems Appl. 19 (2), 383-394, 2011.

[2] Ahmad, B. and Nieto, J. J. Anti-periodic fractional boundary value problems, Comput. Math. Appl. 62, (3) 1150-1156, 2011.

[3] Ahmad, B. and Nieto, J. J. Existence of solutions for impulsive anti-periodic boundary value problems of fractional order, Taiwanese J. Math. 15 (3), 981-993, 2011.

[4] Ahmad, B. and Nieto, J. J. and Johnatan, P. Some boundary value problems of fractional differential equations and inclusions, Comput. Math. Appl. 62 (3), 1238-1250, 2011.

[5] Ahmad, B. and Nieto, J. J. Anti-periodic fractional boundary value problems with nonlinear term depending on lower order derivative, Fract. Calc. Appl. Anal. 15, (3), 451-462, 2012.

[6] Ahmad, B. and Sivasundaram, S. Existence results for nonlinear impulsive hybrid boundary value problems involving fractional differential equations, Nonlinear Anal. Hybrid Syst. 3 (3), 251-258, 2009.

[7] Akhmet, M. Principles of discontinuous dynamical systems (Springer, New York, 2010).

[8] Baĭnov, D. D. and Simeonov, P. S. Systems with impulse effect, Ellis Horwood Series: Mathematics and its Applications (Ellis Horwood Ltd., Chichester, 1989).

[9] Borg, G. On a Liapounoff criterion of stability, Amer. J. Math. 71 67-70, 1949.

[10] Cheng, S. S. Lyapunov inequalities for differential and difference equations, Fasc. Math. 304 (23), 25-41, 1991.

[11] Coppel, W. A. Disconjugacy, Lecture Notes in Mathematics, 220 (Springer-Verlag, BerlinNew York, 1971).

[12] Eloe, P. and Neugebauer, J. T. Conjugate points for fractional differential equations, Fract. Calc. Appl. Anal. 17 (3), 855-871, 2014.

[13] Ferreira, R. A. C. A Lyapunov-type inequality for a fractional boundary value problem, Fract. Calc. Appl. Anal. 16 (4), 978-984, 2013.

[14] Ferreira, R. A. C. On a Lyapunov-type inequality and the zeros of a certain Mittag-Leffler function, J. Math. Anal. Appl. 412 (2), 1058-1063, 2014.

[15] Guseinov, G. S. and Zafer, A. Stability criterion for second order linear impulsive differential equations with periodic coefficients, Math. Nachr. 281 (9), 1273-1282, 2008.

[16] Hartman, P. Disconjugate nth order differential equations and principal solutions, Bull. Amer. Math. Soc. 74 125-129, 1968.

[17] Hartman, P. Ordinary differential equations, Classics in Applied Mathematics 38 (Society for Industrial and Applied Mathematics (SIAM), Philadelphia, PA, 2002).

[18] Jleli, M. and Ragoub L. and Samet B. A Lyapunov-type inequality for a fractional differential equation under a Robin boundary condition, J. Funct. Spaces 2015 Art. ID 468536, 5 pp, 2015.

[19] Jleli, M. and Samet, B. Lyapunov-type inequalities for a fractional differential equation with mixed boundary conditions, Math. Inequal. Appl. 18 (2), 443-451, 2015. 
[20] Jleli, M. and Samet, B. Lyapunov-type inequalities for fractional boundary-value problems, Electron. J. Differential Equations 2015 (88), 1-11, 2015.

[21] Kayar, Z. and Zafer, A. Impulsive boundary value problems for planar Hamiltonian systems, Abstr. Appl. Anal. 2013 Art. ID 892475, 6 pp, 2013.

[22] Kilbas, A. A. and Srivastava, H. M. and Trujillo, J. J. Theory and applications of fractional differential equations, North-Holland Mathematics Studies 204 (Elsevier Science B.V., Amsterdam, 2006).

[23] Lakshmikantham, V. and Baĭnov, D. D. and Simeonov, P. S. Theory of impulsive differential equations, Series in Modern Applied Mathematics 6 (World Scientific Publishing Co. Inc., Teaneck, NJ, 1989).

[24] J. Levin, A. The non-oscillation of solutions of the equation $x^{(n)}+p_{1}(t) x^{(n-1)}+\cdots+$ $p_{n}(t) x=0$, Uspehi Mat. Nauk. 24 (2), 43-96, 1969.

[25] Liapounoff, A. Problème général de la stabilité du mouvement, Annals of Mathematics Studies 17 (Princeton University Press, Princeton, N. J., 1947).

[26] Miller, K. S. and Ross, B. An introduction to the fractional calculus and fractional differential equations, A Wiley-Interscience Publication, (John Wiley \& Sons, Inc., New York, 1993).

[27] Nehari, Z. Green's functions and disconjugacy, Arch. Rational Mech. Anal. 62 (1), 53-76, 1976.

[28] Podlubny, I. Fractional differential equations, Mathematics in Science and Engineering 198 (Academic Press, Inc., San Diego, CA, 1999).

[29] Rong, J. and Bai, C. Lyapunov-type inequality for a fractional differential equation with fractional boundary conditions, Adv. Difference Equ. 2015 Art. ID 82, 10 pp, 2015.

[30] Sabatier, J. and Agrawal, O. P. and Tenreiro Machado, J. A. Advances in fractional calculus. Theoretical developments and applications in physics and engineering, (Springer, Dordrecht, 2007).

[31] Samol̆lenko, A. M. and Perestyuk, N. A. Impulsive differential equations, World Scientific Series on Nonlinear Science. Series A: Monographs and Treatises 14 (World Scientific Publishing Co. Inc., River Edge, NJ, 1995).

[32] Tian, Y and Bai, Z. Existence results for the three-point impulsive boundary value problem involving fractional differential equations, Comput. Math. Appl. 59 (8), 2601-2609, 2010.

[33] Tiryaki, A. Recent developments of Lyapunov-type inequalities, Adv. Dyn. Syst. Appl. 5 (2), 231-248, 2010.

[34] Wang, G. and Ahmad, B. and Zhang, L. Some existence results for impulsive nonlinear fractional differential equations with mixed boundary conditions, Comput. Math. Appl. 62 (3), 1389-1397, 2011.

[35] Wang, F. and Liu, Z. Anti-periodic fractional boundary value problems for nonlinear differential equations of fractional order, Adv. Difference Equ. 2012 (116), 12 pp, 2012.

[36] Wang, J. R. and Zhou, Y. and Fečkan, M. On recent developments in the theory of boundary value problems for impulsive fractional differential equations, Comput. Math. Appl. 64 (10), 3008-3020, 2012.

[37] Zhang, S. Existence of solution for a boundary value problem of fractional order, Acta Math. Sci. Ser. B Engl. 26 (2), 220-228, 2006.

[38] Zhang, L. and Wang, G. Existence of solutions for nonlinear fractional differential equations with impulses and anti-periodic boundary conditions, Electron. J. Qual. Theory Differ. Equ. 2011 (7), 11 pp, 2011

[39] Zhang, L. and Wang, G. and Song, G. On mixed boundary value problem of impulsive semilinear evolution equations of fractional order, Bound. Value Probl. 2012 (17), 8 pp, 2012.

[40] Zhao, K. and Gong, P. Positive solutions for impulsive fractional differential equations with generalized periodic boundary value conditions, Adv. Difference Equ. 2014 (255 ), 19 pp, 2014.

[41] Zhou, J. and Feng, M. Green's function for sturm-liouville-type boundary value problems of fractional order impulsive differential equations and its application, Boundary Value Problems 2014 (69), 1-21, 2014. 
[42] Zhou, W. X. and Liu, X. and Zhang, J.-G. Some new existence and uniqueness results of solutions to semilinear impulsive fractional integro-differential equations, Adv. Difference Equ. 2015 (38), 16 pp, 2015 
